\title{
Automated Wildfire Detection Through Artificial Neural Networks
}

\author{
Jerry Miller (NASA), Kirk Borne (GMU), Brian Thomas (UMD), $\searrow$ \\ Zhenping Huang (UMD), and Yuechen Chi (GMU)
}

\begin{abstract}
Wildfires have a profound impact upon the biosphere and our society in general. They cause loss of life, destruction of personal property and natural resources and alter the chemistry of the atmosphere. In response to the concern over the consequences of wildland fire and to support the fire management community, the National Oceanic and Atmospheric Administration (NOAA), National Environmental Satellite, Data and Information Service (NESDIS) located in Camp Springs, Maryland gradually developed an operational system to routinely monitor wildland fire by satellite observations. The Hazard Mapping System, as it is known today, allows a team of trained fire analysts to examine and integrate, on a daily basis, remote sensing data from Geostationary Operational Environmental Satellite (GOES), Advanced Very High Resolution Radiometer (AVHRR) and Moderate Resolution Imaging Spectroradiometer (MODIS) satellite sensors and generate a 24 hour fire product for the conterminous United States. Although assisted by automated fire detection algorithms, NOAA has not been able to eliminate the human element from their fire detection procedures. As a consequence, the manually intensive effort has prevented NOAA from transitioning to a global fire product as urged particularly by climate modelers. NASA at Goddard Space Flight Center in Greenbelt, Maryland is helping NOAA more fully automate the Hazard Mapping System by training neural networks to mimic the decision-making process of the fire analyst team as well as the automated algorithms.
\end{abstract}

Two years ago, the Computing, Information and Communications Technology (CICT), Program operating out of the Ames Research Center in Moffett Field, California, provided funding for the research effort to get underway. A team of government and (ultimately) University personnel were assembled with the intent of applying artificial intelligence techniques to NOAA's automation problem. NASA began archiving satellite imagery from GOES, AVHRR and MODIS satellite sensors in the summer of 2003. Three spectral channels for each of 3 science instruments were provided by NOAA NESDIS by uploading to a NASA computer within the Information Systems Division at Goddard Space Flight Center. The following spectral bands, being only a subset of those available from each instrument, were found to be the most useful in fire identification by NESDIS: MODIS channels $1(0.66 \mu \mathrm{m}), \mathbf{2}(0.86 \mu \mathrm{m}), \mathbf{2 2}(3.96$ $\mu \mathrm{m})$; AVHRR channels $1(0.66 \mu \mathrm{m}), 2(0.91 \mu \mathrm{m}), \mathbf{3 b}(3.7 \mu \mathrm{m})$, and GOES channels 1 $(0.62 \mu \mathrm{m}), 2(3.9 \mu \mathrm{m}), 4(10.7 \mu \mathrm{m})$. Both reflectance and brightness temperature were scaled by NESDIS to a range of $0-255$.

A good deal of thought, time and attention went into the composition of adequate neural 
network training sets. Early attempts included time and geographic location parameters in addition to spectral information but due to subsequent difficulties in obtaining convergence, ultimately only the spectral information was used. The original guiding principle in training set composition was to use NOAA's ASCII data-formatted fire product, which identifies hotspots by geographic coordinates, to locate fires within satellite imagery, then extract 3-band pixel information at these points.

Using a software package called Environment for Visualizing Images (ENVI), geographic coordinates were converted to pixel row and column coordinates for a particular image being processed through a series of ENVI function calls embedded in IDL code. When examining fires using ENVI in visual mode however it was found that fires were not in the precise location where the geographic coordinates placed them, being offset possibly by several pixels from their expected location. Considering the 1 kilometer spatial resolution of MODIS and AVHRR, the offset error might have been 2 or 3 kilometers but for GOES data in the thermal band (4 KM resolution) the error could have been as much as 12 kilometers. This offset was attributed to 3 sources: spacecraft navigation errors, the inherent tolerances within NOAA software and operational errors in the point-and-click method of a Fire Analyst identifying fire locations with a mouse.

One of the best clues for identifying wildfires that NOAA Fire Analysts employ is to visually inspect the 4 micron band for dark spots within NESDIS post-processed satellite imagery. NOAA software has been written in such a way that brightness temperatures assume the lowest intensity values for the hottest fires (as a means of highlighting them). In order to extract fire signatures from satellite imagery, our software performed a local minima search for the hottest pixels using the approximate location specified by the ASCII data fire product then collected spectral information around that image coordinate in all 3 bands. Three different methods to characterize a fire across 3 spectral bands were investigated: as a single pixel at an instantaneous point of time, a pixel time series demonstrating the time evolution of a fire throughout the day and as a pixel array at an instantaneous point in time. The first two techniques had mixed results in achieving neural network convergence, however the third, a spatial technique consisting of $7 \times 7$ pixel arrays with the hottest part of the fire as the central pixel, was successful.

Three bands of $7 \times 7$ pixel arrays, formatted as 147 element vectors determined the number of network input nodes while the number of hidden nodes was initially determined by the rule-of-thumb to start with the square root of the sum of the inputs and outputs, i.e.12. Even with some experimentation though, the number of hidden nodes did not vary much from the initial value. A single output node was required to discriminate between the 2 classes. The resulting 147-10-1 feedforward backpropagation neural network was then used for training and testing with MATLAB's Neural Network Toolbox.

Thousands of sample fire and non-fire patterns were extracted from satellite imagery for each sensor. For MODIS, the data set consisted of 25,713 samples - the ratio of fires to non-fires being approximately $1: 1$. A variation of the cross-validation technique was employed for training and testing. Total available patterns were divided into 4 quarters, 
each being representative of the entire data set. Training samples constituted $1 / 2$ of the total number of patterns with $1 / 4$ relegated to a validation set and $1 / 4$ a test set resulting in 3 disjoint data sets. Batch training using the Gradient Descent with Momentum algorithm was selected from a suite of available MATLAB routines and Early Stopping was employed to prevent overfitting. During training, the mean squared error (MSE) on the validation set was monitored. Training was automatically halted when validation MSE began to rise. Testing then progressed with data that had not been seen by the neural network during the training phase.

Neural network classification results were initially formulated as error matrices from which a statistical analysis was derived. Since only a 2-class system was involved (fires and non-fires) empirical data consisted of: true positives (TP), true negatives (TN), false positives (FP) and false negatives (FN). Four measures of classification accuracy in terms of these parameters are shown below for the MODIS sensor:

$\begin{array}{lll}\text { Overall Accuracy } & (\mathrm{TP}+\mathrm{TN}) /(\mathrm{TP}+\mathrm{TN}+\mathrm{FP}+\mathrm{FN}) & =92.3615 \% \\ \text { Producer's Accuracy (fire) } & \mathrm{TP} /(\mathrm{TP}+\mathrm{FN}) & =89.9112 \% \\ \text { Producer's Accuracy (nonfire) } & \mathrm{TN} /(\mathrm{FP}+\mathrm{TN}) & =94.7192 \% \\ \text { User's Accuracy (fire) } & \text { TP/(TP+FP) } & =94.2468 \% \\ \text { User's Acuracy (nonfire) } & \mathrm{TN} /(\mathrm{TN}+\mathrm{FN}) & =90.7045 \%\end{array}$

Generally, a high degree of classification accuracy was achieved for the MODIS and AVHRR sensors while classification of GOES image data performed relatively poorly. This was attributed to the accuracy of the science instruments themselves as well as the refinement in fire detection algorithms which followed the earlier GOES methods. The original intent of the project was to develop a single neural network that could process sensor data from all 3 instruments, MODIS, AVHRR and GOES, perform fire classification at least as well as the automated algorithms and human fire analysts currently achieve and be incorporated into NOAA's operational Hazard Mapping System to reduce the amount of manual intervention. However our research has shown that there was insufficient temporal and spatial overlap between the 3 sensors to process image data with a single network. Even excepting this problem, the extreme size of the network, exacerbated by $7 \times 7$ pixel arrays to characterize fire patterns, would have made training difficult or impossible for our host platform. Our work showed that dividing processing between 3 independent networks was the practical solution and that GOES data should probably not be processed at all by the neural network because of the low classification accuracy. Some improvement in classification accuracy is believed likely however by incorporating in the training process additional generalization techniques such as Modified Performance Function and Bayesian Regularization.

Jerry Miller is a Computer Engineer and Researcher with NASA at Goddard Space Flight Center in Greenbelt, Maryland. He specializes in software technology development in support of the Earth Sciences. In the past he has been involved in investigations which dealt with cloud cover detection, atmospheric correction and vectorborne disease forecasting. Mr. Miller resides in Columbia, Maryland with his wife and very rare, silver-gray, miniature poodle. 